University of Nebraska - Lincoln

DigitalCommons@University of Nebraska - Lincoln

Ralph Skomski Publications

Research Papers in Physics and Astronomy

2009

Dzyaloshinski-Moriya Micromagnetics of Magnetic Surface Alloys

Ralph Skomski

University of Nebraska-Lincoln, rskomski2@unl.edu

Jan Honolka

Max-Planck-Institut für Festkörperforschung, honolka@fzu.cz

S. Bornermann

Ludwig-Maximilians Universität München, Geschwister-Scholl-Platz 1, 80539 München, Germany

H. Ebert

Ludwig-Maximilians Universität München, Geschwister-Scholl-Platz 1, 80539 München, Germany

Axel Enders

University of Nebraska-Lincoln, a.enders@me.com

Follow this and additional works at: https://digitalcommons.unl.edu/physicsskomski

Part of the Physics Commons

Skomski, Ralph; Honolka, Jan; Bornermann, S.; Ebert, H.; and Enders, Axel, "Dzyaloshinski-Moriya Micromagnetics of Magnetic Surface Alloys" (2009). Ralph Skomski Publications. 60.

https://digitalcommons.unl.edu/physicsskomski/60

This Article is brought to you for free and open access by the Research Papers in Physics and Astronomy at DigitalCommons@University of Nebraska - Lincoln. It has been accepted for inclusion in Ralph Skomski Publications by an authorized administrator of DigitalCommons@University of Nebraska - Lincoln. 


\title{
Dzyaloshinski-Moriya micromagnetics of magnetic surface alloys
}

\author{
R. Skomski, ${ }^{1, a)}$ J. Honolka, ${ }^{2}$ S. Bornemann, ${ }^{3}$ H. Ebert, ${ }^{3}$ and A. Enders ${ }^{1}$ \\ ${ }^{1}$ Department of Physics and Astronomy and NCMN, University of Nebraska, Lincoln, Nebraska 68588, USA \\ ${ }_{2}^{2}$ Max-Planck-Institut für Feskörperforschung, Heisenbergstraße 1, 70569 Stuttgart, Germany \\ ${ }^{3}$ Ludwig-Maximilians Universität München, Geschwister-Scholl-Platz 1, 80539 München, Germany
}

(Presented 13 November 2008; received 29 August 2008; accepted 9 November 2008; published online 12 March 2009)

\begin{abstract}
The magnetism of magnetic surface alloys such as $\mathrm{Fe}-\mathrm{Pt}$ on $\mathrm{Pt}(997)$ has been investigated by micromagnetic model calculations. A unique feature of some of these systems is that interatomic exchange, magnetocrystalline anisotropy, and Dzyaloshinski-Moriya (DM) interactions are all of comparable magnitude, of the order of $0.5 \mathrm{meV}$. This leads to an intriguing nanoscale interplay between ordinary magnetization states and noncollinear spin structures. We identify two cases where the latter dominate. First, for sufficiently strong DM interaction, the zero-field spin structures change from uniform to a canted state with an incommensurate wave vector. Second, a similar transition occurs for weak DM interaction when the reverse external field approaches the nucleation field. (C) 2009 American Institute of Physics. [DOI: 10.1063/1.3068632]
\end{abstract}

\section{INTRODUCTION}

The spin orientation and magnetic hysteresis of most magnetic materials are determined by magnetocrystalline anisotropy, as epitomized by the first uniaxial anisotropy constant $K_{1}$. Dzyaloshinski-Moriya $(\mathrm{DM})$ interactions ${ }^{1,2}$ are of comparable magnitude but rarely considered in micromagnetism ${ }^{3,4}$ because they require crystals with broken inversion symmetry and directly compete with interatomic exchange. Inversion symmetry is broken in substances such as $\alpha-\mathrm{Fe}_{2} \mathrm{O}_{3}$, but most bulk magnets of interest in magnetism are inversion symmetric. More importantly, the cross product in the DM interaction

$$
E=\mathbf{D}_{i j} \cdot \mathbf{S}_{i} \times \mathbf{S}_{j}
$$

favors a perpendicular spin orientation between neighboring spins $i$ and $j$, but $D$ tends to be much smaller than the interatomic exchange $J$. The latter involves the scalar product $\mathbf{S}_{i} \cdot \mathbf{S}_{j}$ and favors parallel (or antiparallel) spins with $\mathbf{S}_{i} \times \mathbf{S}_{j}$ $=0$. Since $J \gg D$ for most materials, changes of the spin direction due to DM interactions are rather small, typically $1^{\circ}$ or less. Similar arguments apply to spin glasses, ${ }^{5}$ magnetic nanostructures, ${ }^{6}$ and surfaces. ${ }^{7}$

However, the situation is different in some nanostructures, such as Fe-Pt surface alloys, where interatomic exchange, magnetocrystalline anisotropy, and DM interactions are all of comparable magnitude. Such alloys can be produced by submonolayer Fe deposition on $\mathrm{Pt}(997)$ at $525 \mathrm{~K}^{8}{ }^{8}$ The relative strength of the DM interactions in these structures has two reasons. First, the DM interactions (and magnetocrystalline anisotropy) are relativistic phenomena involving spin-orbit coupling, and the spin-orbit coupling parameter is about $0.5 \mathrm{eV}$ for heavy transition metals such as $\mathrm{Pt}$, as compared to about $0.05 \mathrm{eV}$ for the late iron-series transition metals. Note that this pronounced spin-orbit coupling is also exploited in some other thin-film nanostructures

${ }^{\text {a)} E l e c t r o n i c ~ m a i l: ~ r s k o m s k i @ n e b . r r . c o m . ~}$ with high anisotropy, such as Co on Pt. ${ }^{9}$ Second, the atomic environment of the $\mathrm{Fe}$ and $\mathrm{Pt}$ surface atoms yields a strongly reduced interatomic exchange for some structures.

The focus of the present paper is on the effect of the DM interactions on the micromagnetic spin structure. As discussed elsewhere, ${ }^{6,10}$ exchange energies per atom are usually much larger than anisotropy energies per atom, which is the origin of nanoscale magnetization features such as domains and domain walls. We will see that DM interactions compete with traditional micromagnetics in a nontrivial way and yield additional complexity.

\section{MAGNETIC SURFACE ALLOYS}

Recent progress in surface science and nanotechnology has made it possible to create structures with a wide range of interactions and anisotropies. Figure 1 shows two types of Fe-Pt surface alloys, namely, $2 \times 1$ and $2 \times 2$ surface structures. The alloys, as well as a variety of disordered surface alloys, can be prepared by Fe deposition onto $\mathrm{Pt}(997)$ substrates, and the structure depends on deposition conditions and Fe coverage. The use of $\mathrm{Pt}(997)$ has been motivated by the high regularity of the step periodicity, with a step-step distance of $2 \mathrm{~nm} .{ }^{11}$ Below $450 \mathrm{~K}$, a pure $\mathrm{Fe}$ adlayer grows

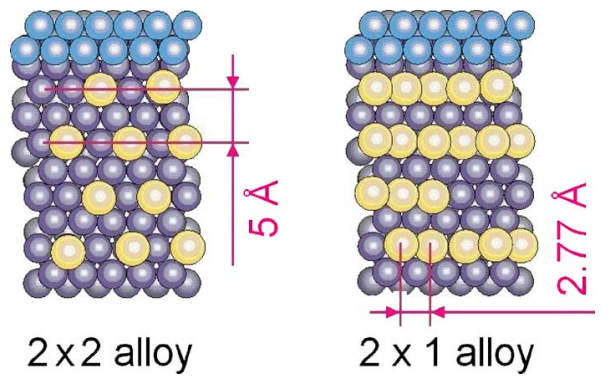

FIG. 1. (Color online) Schematic structures of Fe-Pt surface alloys. The 2 $\times 2$ and $2 \times 1$ structures correspond to iron-poor $\left(\mathrm{Fe}_{25} \mathrm{Pt}_{75}\right)$ and equiatomic $\left(\mathrm{Fe}_{50} \mathrm{Pt}_{50}\right)$ coverages. The $1 \times 1$ and $2 \times 1$ structures correspond to equiatomic $\left(\mathrm{Fe}_{50} \mathrm{Pt}_{50}\right)$ and iron-poor $\left(\mathrm{Fe}_{25} \mathrm{Pt}_{75}\right)$ coverages. 
initially by step decoration, followed by step-flow growth resulting in Fe stripes at submonolayer coverage. Between 500 and $550 \mathrm{~K}$ an Fe-Pt surface alloy is formed by intermixing of Fe with the topmost Pt layer. ${ }^{8}$ The surface alloys grow from the step edges: offering less $\mathrm{Fe}$ leads to an $\mathrm{Fe}_{25} \mathrm{Pt}_{75}$ surface alloy which is confined to the area around the step edges. Details of the fabrication of the surface alloys and of their investigation using experimental methods, especially $\mathrm{X}$-ray magnetic circular dichroism (XMCD), scanning tunneling microscopy (STM) and magnetometry (XMCD, STM, magnetometry) and Koringa-Kohn-Rostoker (KKR) firstprinciples calculations will be published elsewhere. ${ }^{12,13}$

The surface alloys are magnetically ordered at $12 \mathrm{~K}$ and exhibit coercivities of up to $0.71 \mathrm{~T}$ depending on surface stoichiometry and field direction. The predicted induced moments are $0.27 \mu_{B}$ /atom for Pt in a layer of $2 \times 1 \mathrm{FePt}$ on $\mathrm{Pt}(111)$ and $0.21 \mu_{B} /$ atom for Pt when the corresponding alloy layer is statistically disordered. For the first layer of the underlying $\mathrm{Pt}(111)$ substrate we obtain Pt moments of about $0.2 \mu_{B}$ and $0.18 \mu_{B}$. The Fe moment is about $3.2 \mu_{B}$ per atom. These values are not surprising and fall in the range expected for Fe-Pt based structures. ${ }^{14-16}$

The $2 \times 1$ structures exhibit interesting magnetic properties. The Fe atoms are predicted to have a strong ferromagnetic coupling within an Fe chain $(30 \mathrm{meV}$ between nearest neighbor atoms), but the mutual coupling between two $\mathrm{Fe}$ chains is quite small, about $0.5 \mathrm{meV}$ per atom. If the latter value was the only consideration, the Curie temperature would be only about $5 \mathrm{~K}$, in contrast to the observed magnetic order at and probably above $12 \mathrm{~K}$, but the strong intrachain exchange greatly enhances the number of atoms involved in the interchain interaction and explains the observed ferromagnetism. This situation is actually similar to that encountered in low-dimensional ferromagnets with residual three-dimensional exchange coupling. ${ }^{17}$

While the exchange coupling is rather rigid along each $2 \times 1$ chain, neighboring chains are only loosely coupled, and the coupling energy of $0.3 \mathrm{meV}$ per atom is comparable to the magnetocrystalline anisotropy energies of the system $(-1.06$ to $+0.35 \mathrm{meV}$ per atom depending on measurement direction and degree of disorder) and to the DM interaction, which is of the same order of magnitude. This leaves us with the intriguing picture of a magnet where the DM interaction is comparable to both exchange and anisotropy.

\section{MODEL AND CALCULATION}

To see how the DM interactions affect the micromagnetic spin structure, we consider a DM vector pointing in the $y$ direction, $\mathbf{D}=D \mathbf{e}_{y}$, competing with $c$-axis anisotropy in the $z$ direction $\left(K_{1}=0\right)$. If the magnetocrystalline anisotropy was the only consideration, then the magnetization would point in the $\pm z$ directions, but the DM favors spin misalignment in the $x-z$ plane, that is, for $\left(\mathbf{S}_{i} \times \mathbf{S}_{j}\right)_{y} \neq 0$. Let us position the atoms at $x_{i}=i a$ and write the magnetization as $\mathbf{M}_{i}$ $=M_{o}\left(\cos \theta_{i} \mathbf{e}_{z}+\sin \theta_{i} \mathbf{e}_{x}\right)$. With $\theta_{i+1}=\theta_{i}+\partial \theta / \partial x$ we obtain after a short calculation

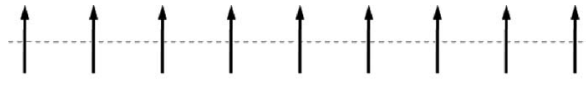

(a)

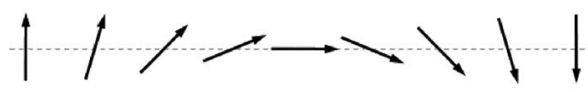

(b)

FIG. 2. Competing spin structures in the systems with broken inversion symmetry: (a) uniform or Stoner-Wohlfarth mode and (b) noncollinear spin structure caused by DM interactions.

$$
\mathbf{D} \cdot\left(\mathbf{M}_{i} \times \mathbf{M}_{i+1}\right)=D M_{o}^{2} a \frac{\partial \theta}{\partial x} .
$$

Together with the Zeeman, exchange, and anisotropy contributions, the energy density is

$$
\eta=A\left(\frac{\partial \theta}{\partial x}\right)^{2}+\frac{D M_{o}^{2}}{a^{2}} \frac{\partial \theta}{\partial x}-K_{1} \cos ^{2} \theta-\mu_{o} M_{s} H \cos \theta .
$$

The functional derivative of $E=\int \eta \mathrm{d} V$ with respect to $\theta$, $\delta E / \delta \theta(x)$, yields the Euler equations

$$
\frac{\partial \eta}{\partial \theta(x)}-\frac{d[\partial \eta / \partial \theta(x)]}{d x}=0 .
$$

However, this equation is only one aspect of the energy minimization. Since $\eta$ is linear in $\partial \eta / \partial \theta(x)$ and $d\left(D M_{o}{ }^{2} / \theta^{2}\right) / d x=0, D$ does not enter Eq. (4). In fact, as we will see in the next paragraph, $D$ affects the spin structure $\theta(x)$ by fixing the integration constants of the differential equation.

Equation (4) is difficult to solves because it establishes a complicated nonlinear problem. However, an approximate solution is obtained by using two trial functions (Fig. 2), namely, the uniform or Stoner-Wohlfarth mode (a), which is the exact solution for $D=0$, and the zero-field isotropic limit, where $K_{1}=H=0$. The latter is obtained by putting the solution of Eq. (4), $\theta(x)=b_{o}+b_{1} x$, into $E=a^{2} \int \eta d x$ and minimizing the result with respect to $b_{o}$ and $b_{1}$. This yields the noncollinear spin structure shown in Fig. 2(b). The periodicity $\lambda$ of the spin structure is incommensurate and given by

$$
\lambda=\frac{4 \pi A a^{2}}{D M_{o}^{2}} \text {. }
$$

For small $D, \lambda$ becomes very large and can, in principle, be observed by macroscopic experimental methods. However, the energy stabilizing this structure, namely, the DM term in Eq. (3), is very small in this limit, and very weak structural or thermal disorder destroys the long-range periodicity.

Comparing the energies between the two modes shown in Fig. 2 yields a sharp transition at

$$
\frac{D^{2} M_{o}^{4}}{A a^{4}}=2 K_{1}\left(1+\frac{H}{H_{a}}\right),
$$

where $H_{a}=2 K_{1} / \mu_{o} M_{s}$ is the anisotropy field. Usually, $D / A$ is a very small quantity, so that DM interactions can be ignored, but there are two notable exceptions. First, $D \sim A a$ 
$\sim K_{1} a^{3}$ for the present surface alloys, so that the present system is a very complicated intermediate case. Second, near the nucleation field, $H=-H_{a}$, the coherent mode becomes very soft and the DM interactions create a macroscopic noncollinearity, at least in the absence of disorder.

It is interesting to note that the modes of Fig. 2 do not mix, that is, the transition between the coherent and noncollinear modes is sharp. To achieve a better description, it is necessary to consider noncollinearities of arbitrary wave vector. Physically, these modes correspond to the interaction of the mode of Fig. 2(b) with micromagnetic features such as domain walls. Domain walls have natural magnetization gradients, and these gradients are enhanced or reduced by the DM interaction depending on the spin structure of the domain wall. The transition between the Stoner-Wohlfarth and noncollinear modes could also be hysteretic, on top of the ordinary hysteresis involving $\uparrow$ and $\downarrow$ configurations.

\section{CONCLUSIONS}

In summary, we have investigated how DzyaloshinskiMoriya (DM) interactions modify the spin structure of magnetic surface alloys such as $2 \times 1$-ordered Fe-Pt. The effect of DM interactions depends on the relative strength of the interatomic exchange. The latter dominates in most magnets and makes DM interactions difficult to observe in systems with nonzero net moment. For weak interatomic exchange, as encountered in the present system, there is a competition between coherent and noncollinear spin structures, and our model predicts a noncollinear spin structure with welldefined wave vector and a sharp transition between coherent rotation and the noncollinear spin structure. A similar transition exists in the vicinity of the nucleation field.

\section{ACKNOWLEDGMENTS}

This research is supported by NSF CAREER (DMR0747704), NSF MRSEC (DMR-0213808), DFG (SPP 1153), and NCMN.

${ }^{1}$ I. J. Dzyaloshinsky, J. Phys. Chem. Solids 4, 241 (1958)

${ }^{2}$ T. Moriya, Phys. Rev. 120, 91 (1960).

${ }^{3}$ R. Skomski, H.-P. Oepen, and J. Kirschner, Phys. Rev. B 58, 11138 (1998).

${ }^{4}$ R. Skomski, A. Kashyap, J. Zhou, and D. J. Sellmyer, J. Appl. Phys. 97, 10B302 (2005)

${ }^{5}$ K.-H. Fischer and A. J. Hertz, Spin Glasses (Cambridge University Press, Cambridge, 1991).

${ }^{6}$ R. Skomski, J. Phys.: Condens. Matter 15, R841 (2003).

${ }^{7}$ M. Bode, M. Heide, K. von Bergmann, P. Ferriani, S. Heinze, G. Bihlmayer, A. Kubetzka, O. Pietzsch, S. Blügel, and R. Wiesendanger, Nature (London) 447, 190 (2007).

${ }^{8}$ T. Y. Lee, S. Sarbach, K. Kuhnke, and K. Kern, Surf. Sci. 600, 3266 (2006).

${ }^{9}$ P. Gambardella, S. Rusponi, M. Veronese, S. S. Dhesi, C. Grazioli, A. Dallmeyer, I. Cabria, R. Zeller, P. H. Dederichs, K. Kern, C. Carbone, and H. Brune, Science 300, 1130 (2003).

${ }^{10}$ R. Skomski, Simple Models of Magnetism (Oxford University Press, Oxford, 2008)

${ }^{11}$ E. Hahn, H. Schief, V. Marsico, A. Fricke, and K. Kern, Phys. Rev. Lett. 72, 3378 (1994).

${ }^{12}$ J. Honolka, T.-Y. Lee, K. Kuhnke, A. Enders, R. Skomski, S. Bornemann, S. Mankovskyy, H. Ebert, K. Fauth, M. Hessler, G. Schütz, A. Buchsbaum, P. Varga, and K. Kern, Phys. Rev. Lett. (in press).

${ }^{13}$ S. Bornemann, R. Skomski, A. Enders, J. Honolka, and H. Ebert (unpublished).

${ }^{14}$ G. Brown, B. Kraczek, A. Janotti, T. C. Schulthess, G. M. Stocks, and D. D. Johnson, Phys. Rev. B 68, 052405 (2003).

${ }^{15}$ A. Kashyap, R. Skomski, A. K. Solanki, Y. F. Xu, and D. J. Sellmyer, J. Appl. Phys. 95, 7480 (2004).

${ }^{16}$ R. Skomski, A. Kashyap, and D. J. Sellmyer, IEEE Trans. Magn. 39, 2917 (2003).

${ }^{17}$ L. J. de Jongh and A. R. Miedema, Adv. Phys. 23, 1 (1974). 